\title{
2006-680: THE ROADMAP OF ARITHMETIC: SUMMING IT UP
}

\section{Andrew Grossfield, Vaughn College of Aeronautics}

Throughout his career Dr. Grossfield, has combined an interest in engineering design and mathematics. He earned a BSEE at the City College of New York. During the early sixties, he obtained an M.S. degree in mathematics at night while working full time during the day, designing circuitry for aerospace/avionics companies. He is licensed in New York as a Professional Engineer and is a member of ASEE, IEEE, SIAM and MAA. Ai207@bfn.org is his e-mail address. 


\title{
The Roadmap of Arithmetic: Summing it up
}

\begin{abstract}
Notice this problem of human consciousness. Ideas and concepts enter our mind one at a time. Usually teachers and authors have the entire course content in their minds before they begin a course or write a text. However, a student can only confront one idea at a time and so the course content is arranged sequentially, one topic after another, one word after another. It is then left to the student to construct in his mind the best arrangement of the material. We must accept that courses and texts are sequentially ordered. Pages are numbered. A student, when confronted by a particularly difficult part of the text, may set the text aside never to see the remainder of the course. The problem is: what is the best arrangement for course material so that a student can visualize the major components of a course at the beginning and subsequently fill in the details?

This paper presents a roadmap in the form of a tree structure (See the chart at the conclusion.) that will allow young students to treat their study of arithmetic as a research project. The roadmap will provide a navigational aid to assist in the exploration of the world of numbers. Historically, the discovery of the periodic table guided scientists in the study of chemistry, indicating what was known and where gaps existed and raising questions about what remained to be explored. The periodic table has been immensely valuable, even though its final form may differ from the table that was originally proposed. This roadmap will serve students similarly in their study of arithmetic.
\end{abstract}

In every well-planned course, only one thing is studied. In arithmetic, the subject is numbers and so the word 'numbers' is placed at the top of the roadmap. At the end of the course, a student should know the kinds, forms, operations, properties and uses of numbers. In our society, this study takes a few years and is often disorganized. It should not be surprising that a young student might become confused and disenchanted. At the end of the course, the strategy should be reviewed so that the student can see what has been learned about numbers and how numbers are used and what remains for future study.

This roadmap suggests that including in the course presentation, a tree structure, giving equal importance to the kinds, operations, forms and properties of numbers, has conceptual advantages. The tree structure separates these concepts but highlights their interaction in the solution of quantitative problems. The visualization of the tree structure together with an understanding of the important concept of forms may aid students in mastering the subject.

The study of numbers is unending and so this roadmap cannot be exhaustive. Still it can provide a framework for introducing the study of numbers. 


\section{What are numbers?}

Numbers are symbols, which can convey information about size, order or position. Their crudest use is as identifiers such as are seen on the backs of football players. In this use, they are never added, subtracted or compared. Numbers used to identify houses as street addresses not only identify but also provide information about the order of the houses on the street and also indicate on which side of the street houses are situated. Numbers are categorized by their algebraic or topological (distance) properties.

\section{Kinds of numbers}

The numbers, which are used to indicate the size of sets of discrete (separate and distinct) objects, are the natural or counting numbers (1,2,3 etc). Children learn the counting numbers and their order in the same way they learn the alphabet, by memorization. Later they learn to add, subtract and multiply the counting numbers. As we know, in the set of counting numbers, it is not meaningful to subtract a larger number from a smaller number. We describe this situation by saying that the set of counting numbers is closed under addition and multiplication but is not closed with respect to subtraction.

The numbers, which indicate not only a discrete amount but also a direction, are called integers and are the signed whole numbers $(0, \pm 1, \pm 2, \pm 3$ etc). The integers may be added, multiplied and subtracted, and the result of the operation will always yield another integer. That is; the set of integers is closed under the operations of addition, multiplication and subtraction. But the set of integers is not closed under division. For example, there is no integer whose value equals to 5 divided by 3 .

The real number system is composed of all the numbers, each of which can be plotted on a straight line. One point is designated as zero. The counting numbers comprise a subset of the real numbers that are conventionally spaced uniformly to the right of zero extending indefinitely. The integers are plotted uniformly with the positive numbers toward the right and the negative numbers toward the left. The real number system is closed under any combination of the basic four arithmetic operations; addition, subtraction, multiplication and/or division (except for division by zero). Sometimes there are advantages to thinking of the numbers as points on a line but there are other times when the distinction between numbers and points should be maintained.

The smallest subset of the real number system, which contains the integers and is closed under the basic four operations (except division by zero) is the set of rational numbers; that is, those

numbers, which can be described by the ratio $\frac{m}{n}$ where $m$ and $n$ represent integers( e.g. $\left.\pm \frac{2}{3}\right)$. It can be shown that an infinite number of rational numbers can be found between any two different rational numbers and therefore a rational number has no immediate predecessor or successor. The rational numbers are not described as discrete. The set of rational numbers is described as dense in the set of real numbers. 
Since the rational numbers are dense, at one time it was believed that all the real numbers were rational. But it was shown not to be so. In particular, the diagonal of a square whose sides have length equal to 1 cannot be expressed as a ratio of integers and therefore cannot be rational. Polynomial equations in a single variable may have real solutions that are not rational. The solutions or roots of these polynomial equations are called algebraic numbers. The set of all real algebraic numbers is closed under the basic four operations (except division by zero). The set of positive algebraic numbers is also closed under the operation of taking roots. The set of algebraic numbers, which includes the rational numbers, is also dense in the set of real numbers.

The set of rational numbers is also closed under the operation of integer powers ( except when the base is zero ) but not fractional powers (or roots). Examples are that while the numbers $2^{2}=4$ and $2^{-2}=\frac{1}{4}$ are rational, the values $2^{(1 / 2)}=\sqrt{2}$ and $2^{(-1 / 2)}=\sqrt{\frac{1}{2}}$ are algebraic numbers.

Students are usually introduced to algebraic numbers, denoted by radicals. Compared to radical notation, fractional exponent notation has conceptual and computational advantages. Compare:

to

$$
{\sqrt[5]{3^{2}}}^{5}=3^{2}=9
$$$$
\left(3^{(2 / 5)}\right)^{5}=3^{(2 / 5) * 5}=3^{2}=9 \text {. }
$$

The multiplication $\frac{2}{5} * 5$ in the exponent produces the answer naturally.

Mathematicians have shown that there exist real numbers that are neither integers, nor rational nor algebraic; that is, numbers which are not the solutions of polynomial equations. One such number is the number that represents the ratio of the circumference of every circle to its diameter. This number is called $\boldsymbol{\pi}$. Another is the number, denoted by $\mathbf{e}$, which appears in the description of natural growth and decay processes. These non-algebraic numbers, which are needed to complete the real number line, are called the transcendental numbers. In summary, the real number system contains as a dense proper subset the algebraic numbers, which contain as a dense proper subset the rational numbers, which in turn, contain the uniformly spaced integers, which contain the counting numbers. 


\section{Number system}

Counting numbers

Integers

Rational numbers

Algebraic numbers

Transcendental numbers

\section{Closure features}

closed under addition, multiplication, counting number exponent

above operations plus subtraction

above operations plus integer exponents, excluding division by zero

While positive algebraic numbers can be represented with rational exponents, negative numbers do not have even roots in the real number system.

All of the remaining real numbers.

Again note: Negative numbers do not have even roots and division by zero is always meaningless and excluded.

Because the numbers that are seen in common use are either integers, fractions or truncated numbers in decimal form or scientific notation, someone could reasonably conclude that transcendental numbers are rare. Such is not the case. While comparison of infinite sets is not obvious, Cantor devised a construction that showed that if an attempt were made to match algebraic numbers one-to-one with the transcendental numbers, the algebraic numbers would be depleted while there were still transcendental numbers remaining. In a course on the theory of measure, a comparison of the sets of the algebraic and transcendental numbers in the interval between zero and one; would reveal that the set of algebraic numbers have measure equal to zero while the measure of the set of transcendental numbers is one. Transcendental numbers are not rare. The transcendental numbers are not observed in common use because it is impossible to write them exactly. Like $\pi$ and e, they must be approximated with rational numbers.

\section{Properties of numbers}

In textbooks, the properties of numbers are described in the laws. The properties of numbers involving the operations of addition, multiplication and powers and the inverses of these operations are called the algebraic properties. The properties of numbers concerning the relations $>, \geq,<$, and $\leq$ are called the order properties. The properties of numbers involving distance between numbers are called the topological properties. Mathematicians study sets that strictly exhibit only the algebraic properties (groups and fields) or order properties (order types and ordinal numbers) or topological properties. Mathematicians also study sets, which have combinations of properties; ordered topologies, topological algebras, etc. In the real number system all of these properties are combined. In the table below the distance between the points a and $\mathrm{b}$ is denoted by $\mathrm{d}(\mathrm{a}, \mathrm{b})$ or $|\mathrm{b}-\mathrm{a}|$. The following table lists some of the laws describing the properties of numbers. 
$\underline{\text { Pure Laws }}$

Algebraic

Order

Distance

$\underline{\text { Laws of combinations }}$

algebra and distance

algebra and order

order and distance

\section{$\underline{\text { Examples }}$}

Commutative addition

Commutative multiplication

Distributive

Multiplication and addition

Exponentiation and multiplication

Expansion/Factoring identities that derive from the above

if $\mathrm{a}>\mathrm{b}$ and $\mathrm{b}>\mathrm{c}$, then $\mathrm{a}>\mathrm{c}$

$\mathrm{d}(\mathrm{x}, \mathrm{x})=0$

if $x \neq y$ then $d(x, y)>0$

$\mathrm{d}(\mathrm{x}, \mathrm{y})=\mathrm{d}(\mathrm{y}, \mathrm{x})$

\section{Examples}

$|a \pm b| \leq|a|+|b|$

If $a \geq 0$ and $b \geq 0$ than $a+b \geq 0 ; \quad x^{2} \geq 0$.

If $\mathrm{a}<\mathrm{b}<\mathrm{c}$ and $\mathrm{d}(\mathrm{a}, \mathrm{b})<\mathrm{d}(\mathrm{a}, \mathrm{c})$.

We should observe that all the algebraic identities such as the expansion/factoring equations, \{e.g. $\left.x^{3}-8=(x-2)\left(x^{2}+2 x+4\right)\right\}$ can be viewed as algebraic properties of numbers. It is amazing that these special equations, the identities, hold true for every number chosen for $\mathrm{x}$.

\section{Forms of numbers}

One advantage of the roadmap is that it highlights the importance of representations of numbers in specific forms. While young students may grasp the concepts of the kinds, operations and properties of numbers, the concept and considerations of forms might be more elusive. The concept of form pervades all areas of mathematical thought, yet it is not promoted at the K-12 or college levels. Many mathematical computations and solution strategies are easily explained in terms of forms and changing forms.

The most important of number forms is the wonderful, compact, positional, decimal representation of numbers as sums of powers of ten. This form allows addition, subtraction and multiplication to be accomplished fairly easily. Following is a table describing the decimal forms for the different kinds of numbers:

Integers: $\quad$ Digits are needed only to the left of the decimal point

Rational numbers: Digits to the right of the decimal point either terminate or repeat periodically, unendingly.

Irrational numbers: Means either algebraic or transcendental numbers -- Digits to the right of the decimal point neither terminate nor repeat periodically. 
The truncation or rounding of the unending sequence of digits in the decimal description of irrational numbers creates difficulties. A calculator can hold only so many digits in its registers. The fractional and rounding approximations of irrational numbers can confuse students. Is $\pi$ $3.14,3.14159$ or $22 / 7$ ? These numbers are all different. Which number should a student use? Which number will a calculator compute or display?

The basic difficulty is that none of these numbers, $3.14,3.14159$ or $22 / 7$ is equal to $\pi$. Subtraction of different approximations of the same irrational number will not produce zero. While modern calculating devices are precise enough for almost all situations, students should be aware that they might obtain surprising results. Note that calculations involving rational numbers that are computed (without truncation) and displayed as fractional forms provide exact solutions.

Some forms are motivated by specific needs. In order to describe very large and very small numbers, scientific notation was developed. Since the rules for manipulating numbers pertain to combination of like forms, the solution of problems in which the numbers are presented in different forms requires the conversion of all the numbers into the most suitable form.

\section{Canonical Forms}

Canonical forms are the special forms where the described object has a unique representation and where special aspects of the object are highlighted. Examples of canonical forms are the prime factorization form of integers or the reduced form of rational numbers. The prime factored form of integers is useful in computing least common multiples and greatest common divisors. The concept of canonical forms pervades algebra and advanced mathematics.

\section{Number Operations}

Of course, students of arithmetic should understand the number operations: addition, subtraction, multiplication, division, powers and roots. Scientific calculators have keys that perform these operations. Some calculators will perform these operations on rational numbers entered in fractional form. Integers and rational numbers expressed in prime factored form can be directly multiplied and divided but addition and subtraction can only be performed indirectly.

The mechanism of addition and subtraction of rational fractions is easily explained with the concept of forms. If the fractions have the same common denominator, adding and subtracting the respective numerators while maintaining the common denominator can accomplish the calculation. A problem arises if the denominators are different. The solution is to convert all the fractions into a form where they have a common denominator, CD. But first a suitable denominator must be found. The strategy of the computation is to use any common multiple of the denominators as the denominator, $\mathrm{CD}$, and express all the fractions in the form with $\mathrm{CD}$ as their denominator. Now the numerators can respectively be added and subtracted. 
As an example, consider:

$$
\frac{2}{3}+\frac{3}{5}-\frac{4}{7}=\frac{2}{3} *(1)+\frac{3}{5} *(1)-\frac{4}{7} *(1)
$$

Use the appropriate form of one (1) that produces

the common denominator, $3 * 5 * 7=105$ :

$$
=\frac{2}{3} * \frac{35}{35}+\frac{3}{5} * \frac{21}{21}-\frac{4}{7} * \frac{15}{15}
$$

Combine the factors to obtain the form suitable for addition and subtraction.

$$
\begin{aligned}
& =\frac{2 * 35}{105}+\frac{3 * 21}{105}-\frac{4 * 15}{105} \\
& =\frac{70+63-60}{105}=\frac{73}{105}
\end{aligned}
$$

\section{Summary}

We all learned arithmetic sequentially from linearly structured texts. A question for consideration is: could a tree topic structure, in providing a strategic overview, be conceptually advantageous for some students compared with any linear structure? Perhaps it could help a student maintain his/her bearings during the years that arithmetic is being studied. This proposed structure could be applied not only to arithmetic, but also to many other mathematics courses including algebra, calculus, linear algebra, multivariable calculus and group theory.

If it is desirable for students to learn to formulate their own strategies of solution for quantitative problems and not just memorize cookbook routines, they will need guides such as this roadmap. With the right roadmap, a student will not only know where he started and where he is going, but he may even enjoy the journey.

\section{References:}

1. Grossfield, Andrew "On the Intrinsic Structure of Calculus" Proceedings of the 1995 ASEE Annual Conference, Session 1265 (311-315)

2. Grossfield, Andrew "What is College Algebra?" Proceedings of the Fall 1998 ASEE Middle Atlantic Section Conference

3. Grossfield, Andrew "Mathematical Forms and Strategies" Proceedings of the 1999 ASEE Annual Conference 4. Grossfield, Andrew "Mathematical Definitions: What is this thing?" Proceedings of the 2000 ASEE Annual Conference 


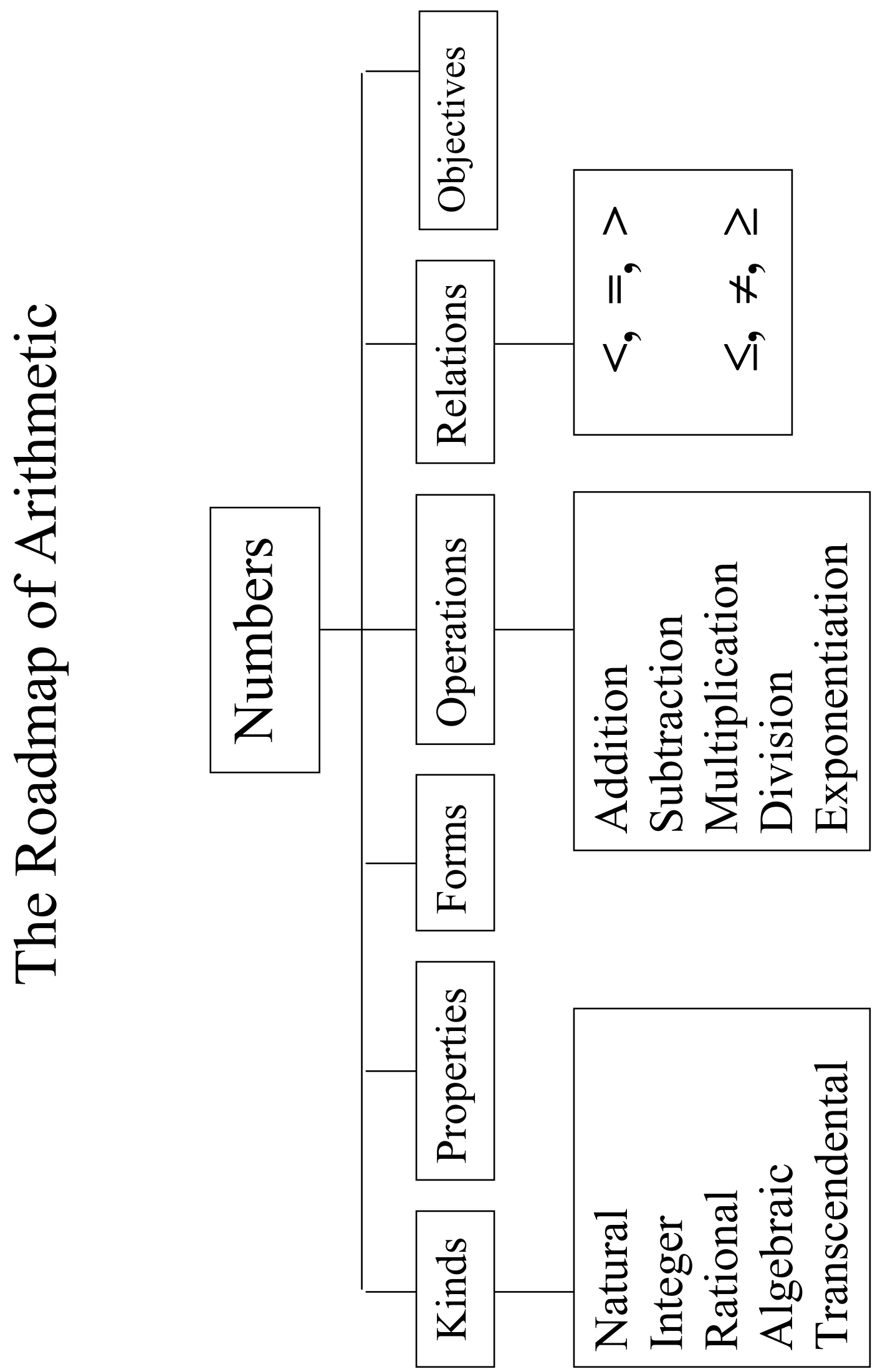

